\title{
Cervical squamous cell carcinoma coexisting with ectopic gastric mucosa treated by endoscopic submucosal dissection: A case report
}

\author{
TOSHIFUMI TSUJI ${ }^{1}$, KEN INOUE $^{2}$, KOICHIRO OMORI $^{1}$, KOHEI OKA ${ }^{1}$, NAOTO IWAI $^{1}$, \\ TASUKU HARA $^{1}$, YUTAKA INADA ${ }^{1}$, TAISHI HARADA ${ }^{3}$, TAKASHI OKUDA ${ }^{1}$, \\ TOSHIYUKI KOMAKI ${ }^{1}$, AKIHIRO NAGATA ${ }^{4}$ and KEIZO KAGAWA ${ }^{1}$
}

\footnotetext{
${ }^{1}$ Department of Gastroenterology and Hepatology, Fukuchiyama City Hospital, Fukuchiyama City, Kyoto 620-8505;

${ }^{2}$ Department of Gastroenterology and Hepatology, Kyoto Prefectural University of Medicine, Kyoto 602-8566;

Departments of ${ }^{3}$ Oncology and ${ }^{4}$ Pathology, Fukuchiyama City Hospital, Fukuchiyama, Kyoto 620-8505, Japan
}

Received April 21,2019; Accepted April 2, 2020

DOI: $10.3892 / \mathrm{mco} .2020 .2037$

\begin{abstract}
A 69-year-old Japanese man underwent an endoscopic submucosal dissection (ESD) for early gastric cancer 2 years prior to admission at Fukuchiyama City Hospital. A follow-up esophagogastroduodenoscopy (EGD) revealed a butterfly-shaped brownish area in the cervical esophagus. A successful endoscopic submucosal dissection of the esophageal lesion was performed for total biopsy. Histopathological analysis of the resected specimen revealed moderately differentiated squamous cell carcinoma of the esophagus, cervical esophagus tumor type 0 -IIb, a tumor diameter of 14x8 mm, T1b pathological findings (invading into submucosa), infiltrative growth $\alpha$, lymphatic invasion $(-)$, venous invasion (-), a horizontal tumor free margin and a vertical tumor free margin. The results demonstrated that the squamous cell carcinoma had invaded into the submucosal layer under the ectopic gastric mucosa. After endoscopic treatment, chemoradiation therapy was administered to the patient.
\end{abstract}

\section{Introduction}

Esophageal cancer is the sixth common cause of cancer associated deaths worldwide (1). It is often difficult to notice esophageal cancer using conventional esophagogastroduodenoscopy (EGD) with white-light imaging (WLI). Many

Correspondence to: Dr Ken Inoue, Department of Gastroenterology and Hepatology, Kyoto Prefectural University of Medicine, 465 Kajii-cho, Kawaramachi-Hirokoji, Kamigyo-ku, Kyoto 602-8566, Japan

E-mail: keninoue71@koto.kpu-m.ac.jp

Abbreviation: ESD, endoscopic submucosal dissection

Key words: squamous cell carcinoma, ectopic gastric mucosa, endoscopic submucosal dissection clinical studies have reported on the diagnostic performance by image-enhanced endoscopy with narrow-band imaging (NBI) (2,3). Moreover, endoscopic surveillance of Barrett's esophagus has become a foundation of the management of esophageal adenocarcinoma, and this trend has accelerated with recent developments in advanced endoscopic imaging and treatment technologies (4).

Endoscopic submucosal dissection is a lower invasive therapy for superficial esophageal cancers. However, esophageal treatment requires more skill than gastric resection because of the technical difficulties related with the narrow location and thin wall in esophagus usually. It is more difficult to distinguish between ectopic gastric mucosa and early cancer, it rarely reported that the case for patient to undergo magnifying endoscopy with NBI and endoscopic submucosal dissection (ESD) for an esophageal adenocarcinoma in the upper thoracic esophagus (5). Therefore, we have reported a first case of squamous cell carcinoma coexisting with ectopic gastric mucosa treated by endoscopic submucosal dissection. I confirmed that the patient provided informed consent for the use of this data with a consent form.

\section{Case report}

A 69-year-old Japanese man underwent an ESD for early gastric cancer 2 years prior to his admission. A follow-up EGD with NBI system showed a butterfly-shaped brownish area (Fig. 1) in the cervical esophagus. The patient's history included hypertension and chronic kidney disease, and his family history included a brother with gastric and colonic cancer. The patient had smoked for 20 years beginning when he was 20 years old.

NBI magnifying endoscopy, revealed that the patient's butterfly-shaped brownish area had type B2 vessels (per the Japanese esophageal Society expansion endoscopic classification (6) in 2/3 of its area (Fig. 2A and B). In addition, $1 / 3$ of this area showed a gastric mucosal pattern (Fig. 2C).

The endoscopic image of the same lesion after spraying with Lugol's solution showed that the lesion remained 


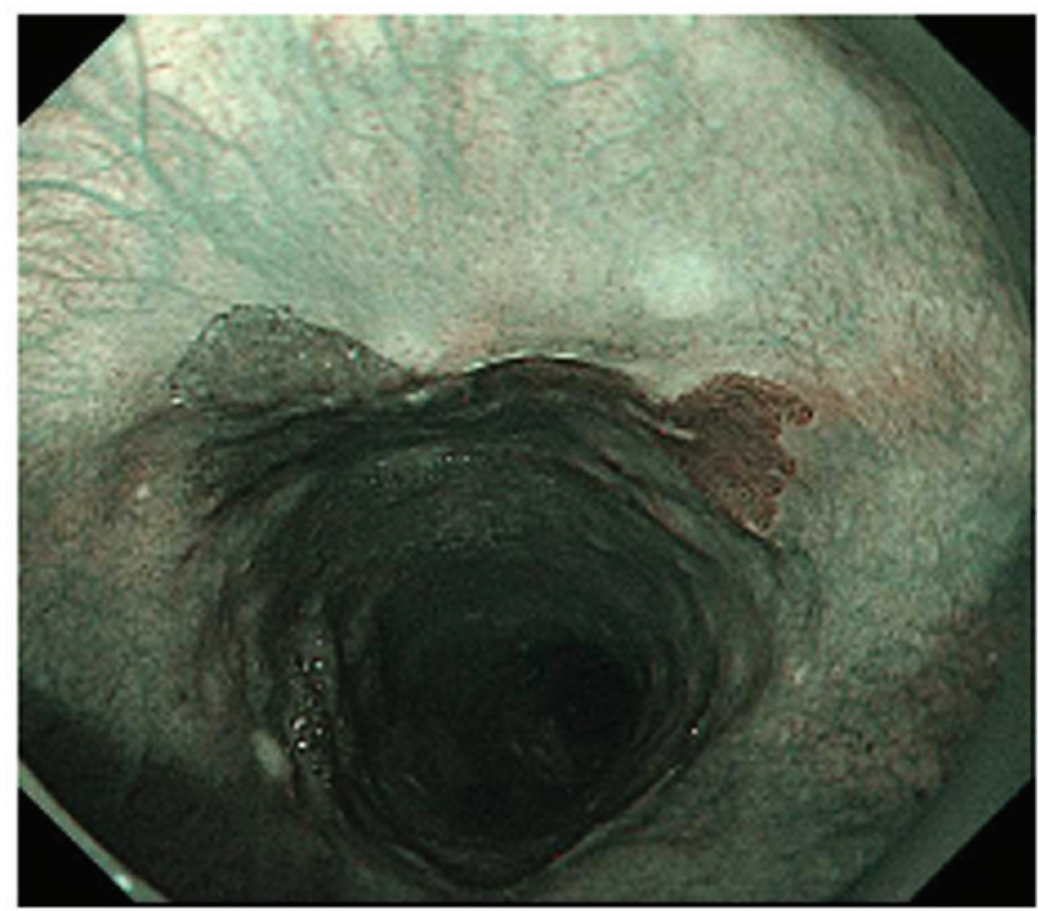

Figure 1. Esophagogastroduodenoscopy with a narrow-band imaging system revealed a $15 \mathrm{~mm}$ butterfly-shaped brownish area at the first endoscopic examination.
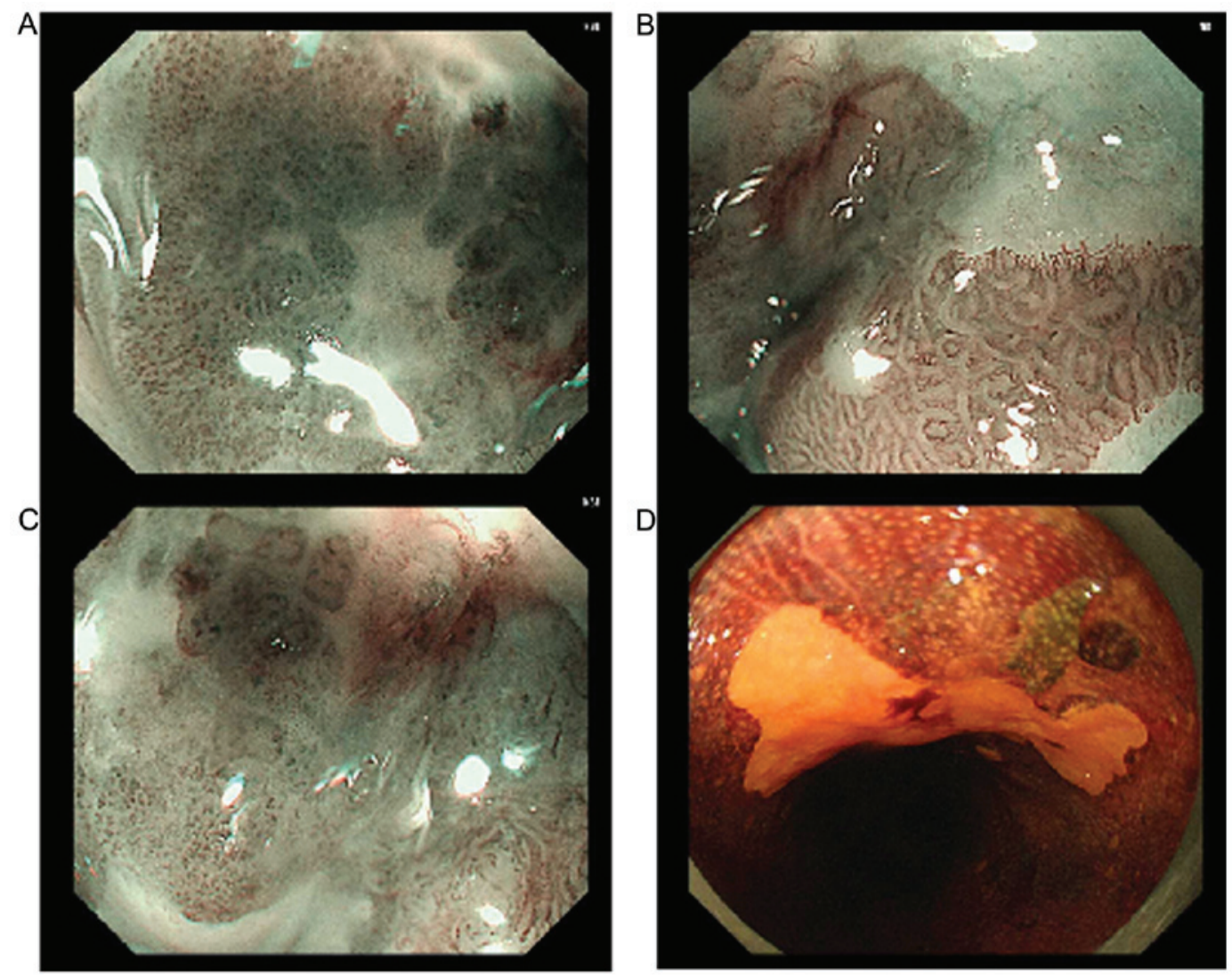

Figure 2. Endoscopic images of esophageal carcinoma. (A) Narrow-band imaging revealed an irregular intra-papillary capillary loop in esophageal mucosa and an irregular microsurface pattern and microvascular pattern in gastric mucosa. (B) Magnifying endoscopy revealed type B2 vessels in 2/3 of this lesion. (C) One-third of this area exhibited a gastric mucosal pattern. (D) Endoscopic image of the unstained lesion after spraying with Lugol's solution.

unstained (Fig. 2D). A squamous cell carcinoma was diagnosed based on the biopsy from the lesion, which revealed type B1-2 vessels.
Because of a localization in cervical esophagus, the surgical operation method will be Pharyngolaryngoesophagectomy. Considering that endoscopic treatment will be more beneficial 


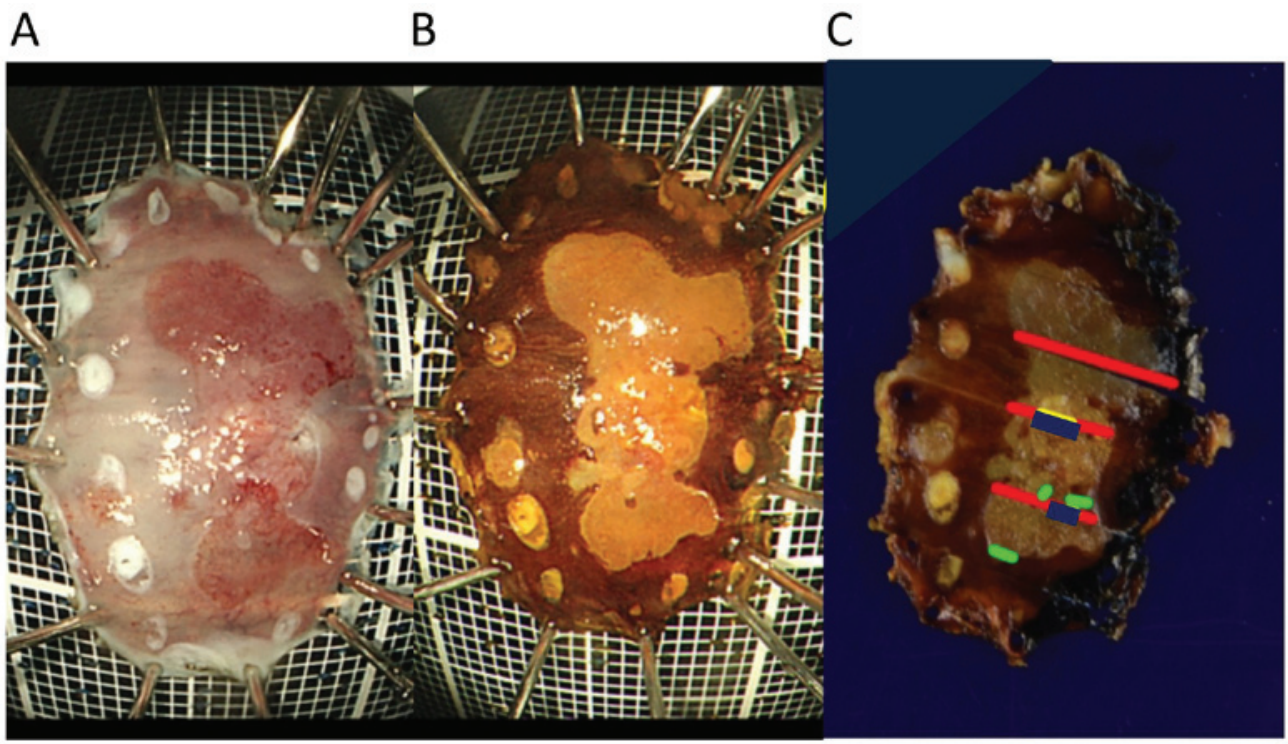

Figure 3. Macroscopic findings. (A) Macroscopic appearance of the resected specimen exhibiting the esophageal cancer as a red lesion. (B) Squamous cell carcinoma unstained in the specimen after spraying with Lugol's solution. (C) The red line demonstrates squamous cell carcinoma of the mucosal layer. The blue line marks the squamous cell carcinoma of the submucosal layer. The green line indicates the ectopic gastric mucosa.

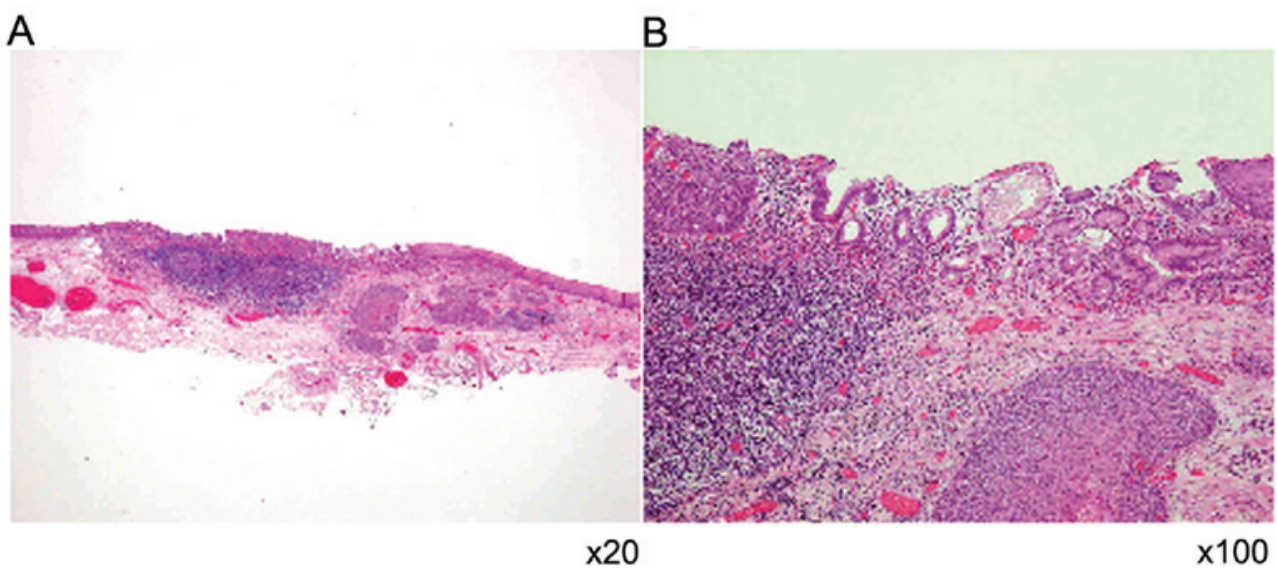

C
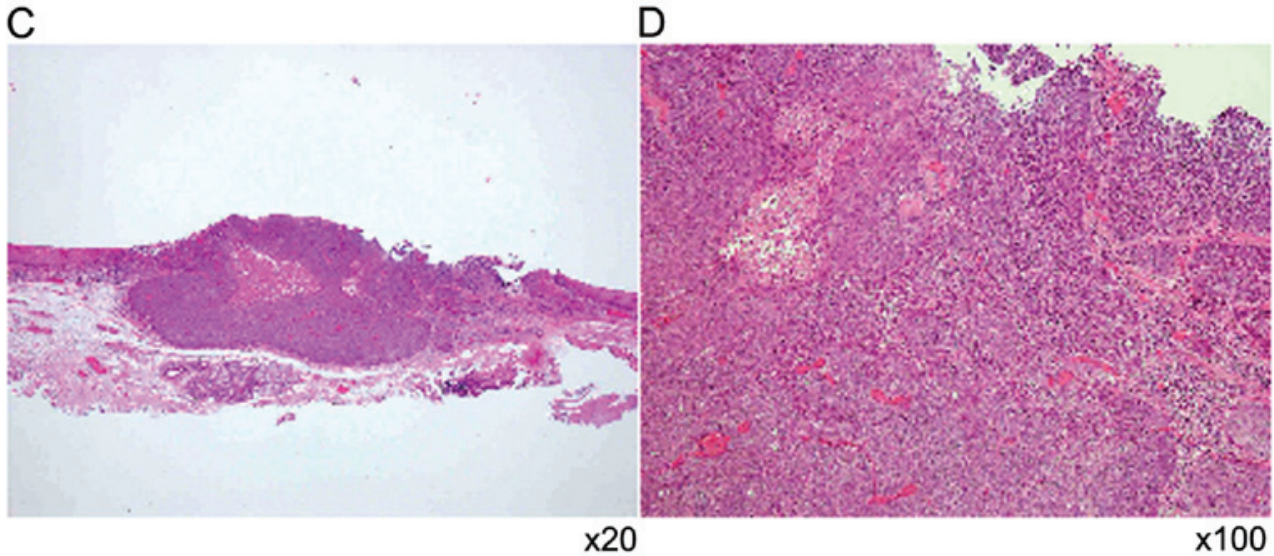

Figure 4. Histological examination of the endoscopically resected specimen. (A) Microscopy revealed that the esophageal lesion was located in the submucosa layer (H\&E staining; magnification, x20). (B) High-magnification image demonstrating tumor invasion into the ubmucosal layer under the ectopic gastric mucosa (H\&E staining; magnification, x100). (C) Esophageal carcinoma invaded deeply into the submucosal layer (H\&E staining; magnification, x20). (D) H\&E staining revealed massive infiltration of esophagus cancer cells (magnification, x100). H\&E, hematoxylin and eosin.

for patient, we performed an endoscopic submucosal dissection (ESD) of the esophageal lesion for the purpose of doing a total biopsy. We completed that endoscopic surgery as planned (Fig. 3A-C). The histopathological analysis of the resected specimen revealed squamous cell carcinoma (moderately differentiated) of the esophagus, Ce, $0-\mathrm{IIb}$, 
14x8 mm, pT1b(SM1), INF $\alpha$, ly0(D2-40), v0(EVG), pHM0, pVM0 (Fig. 4A-D). The squamous cell carcinoma invaded into the submucosal layer under the ectopic gastric mucosa.

\section{Discussion}

We performed an endoscopic submucosal dissection of the cervical esophageal lesion for a total biopsy as planned. The reason why I determined that ESD would be more benefical is a location of esophageal cancer. Because of a localization of cervical esophagus, the operation will be pharyngolaryngoesophagectomy. Needless to say, blood cancer biomarker of squamous cell cancer was normal. Metastatic lesion was not detected in all body CT scan.

The reported frequency of ectopic gastric mucosa of the upper esophagus is $11 \%$ (7). Approximately 30 case reports of adenocarcinoma occurring from ectopic gastric mucosa of the esophagus have been reported since 1950 (8), but our search of the literature revealed no reported case of squamous cell carcinoma neighboring and invading ectopic gastric mucosa. As another rare case, there are a report to undergo ESD for esophageal adenocarcinoma with enteroblastic differentiation arising from ectopic gastric mucosa in the esophagus (9). In our case, the squamous cell carcinoma invaded into the submucosal layer under the ectopic gastric mucosa. From now, it is necessary for us to consider how to be invaded into submucosal layer pathologically. We have thus apparently reported the first case of a patient with squamous cell carcinoma coexisting with ectopic gastric mucosa treated by endoscopic submucosal dissection.

\section{Acknowledgements}

Not applicable.

\section{Funding}

No funding was received.

\section{Availability of data and materials}

The datasets used and/or analyzed during the present study are available from the corresponding author on reasonable request.

\section{Authors' contributions}

TT and KI performed the ESD of the esophageal lesion. KOm and NI aided the endoscopic surgery. KOk and YI, TasH performed white-light and narrow band imaging magnifying endoscopy. TaiH and TO performed an ESD for early gastric cancer. AN performed histological examinations of esophageal cancer tissue. TK and KK supervised treatment. KK wrote the manuscript. All authors read and approved the final manuscript.

\section{Ethics approval and consent to participate}

The present study was approved by the Fukuchiyama City Hospital (Kyoto, Japan). Informed consent was obtained from the patient.

\section{Patient consent for publication}

Informed consent was obtained for publication of patient data.

\section{Competing interests}

The authors declare that they have no competing interests.

\section{References}

1. Bray F, Ferlay J, Soerjomataram I, Siegel RL, Torre LA and Jemal A: Global cancer statistics 2018: GLOBOCAN estimates of incidence and mortality worldwide for 36 cancers in 185 countries: CA Cancer J Clin 68: 394-424, 2018.

2. Gai W, Jin XF, Du R, Li L and Chai TH: Efficacy of narrow-band imaging in detecting early esophageal cancer and risk factors for its occurrence. Indian J Gastroenterol 37: 79-85, 2018.

3. Chiu PWY, Uedo N, Singh R, Gotoda T, Ng EKW, Yao K, Ang TL, Ho SH, Kikuchi D, Yao F, et al: An Asian consensus on standards of diagnostic upper endoscopy for neoplasia. Gut 68: 186-197, 2019.

4. Ishihara R, Goda K and Oyama T: Endoscopic diagnosis and treatment of esophageal adenocarcinoma: Introduction of Japan Esophageal Society classification of Barrett's esophagus. J Gastroenterol 54: 1-9, 2019.

5. Nonaka K, Watanabe M, Yuruki H, Okuda A, Sakurai K, Iyama K and Sasaki Y: Narrow band imaging of adenocarcinoma arising from ectopic gastric mucosa in the upper esophagus. Endoscopy 45 (Suppl 2) UCTN: E112-E113, 2013.

6. Oyama T, Inoue H, Arima M, Momma K, Omori T, Ishihara R, Hirasawa D, Takeuchi M, Tomori A and Goda K: Prediction of the invasion depth of superficial squamous cell carcinoma based on microvessel morphology: Magnifying endoscopic classification of the Japan Esophageal Society. Esophagus 14: 105-112, 2017

7. Weickert U, Wolf A, Schröder C, Autschbach F and Vollmer H: Frequency, histopathological findings, and clinical significance of cervical heterotopic gastric mucosa (gastric inlet patch): A prospective study in 300 patients. Dis Esophagus 24: 63-68, 2011.

8. Paulides E, de Boer NK and Grasman ME: Proximal esophageal cancer missed during esophagogastroduodenoscopy: Should the detection of an inlet patch be added to the quality criteria for upper gastrointestinal endoscopy? Endoscopy 48 (Suppl 1): E273, 2016.

9. Gushima R, Narita R, Shono T, Naoe H, Yao T and Sasaki Y: Esophageal adenocarcinoma with enteroblastic differentiation arising in ectopic gastric mucosa in the cervical esophagus: A case report and literature review. J Gastrointestin Liver Dis 26: 193-197, 2017. International (CC BY-NC-ND 4.0) License. 